performance benefits from wearing CS under these conditions. Limitations of this study include the small number of participants, the lack of female participants, and the short duration of the race.

(Eur J Sport Sci. 2014;14:144-150) F Vercruyssen, C Easthope, T Bernard, et al.

Prepared by Pearlly Ng, MD, University of Utah Wilderness Medicine Fellow, Salt Lake City, UT, USA.

\section{ANTIMICOBIAL AGENTS AND CHEMOTHERAPY}

\section{Efficacy of an Experimental Azithromycin Cream for Prophylaxis of Tick-Transmitted Lyme Disease Spirochete Infection in a Murine Model}

Lyme disease is caused by the spirochete Borrelia burgdorferi and is transmitted by the tick Ixodes scapularis. There are approximately 30,000 reported cases of Lyme disease each year in the United States. Complications from Lyme disease range from mild to life threatening, including rash, arthritis, Bell's palsy, radiculoneuropathy, encephalitis, and cardiac dysfunction. Prophylactic measures, including a vaccine and oral doxycycline, have been attempted with only moderate success, indicating a need for further research.

This study was a randomized controlled murine trial comparing $4 \%$ topical azithromycin with $4 \%$ topical doxycycline for Lyme disease prophylaxis after inoculation via infected tick bite. The primary outcome was B. burgdorferi infection at 1 month after exposure confirmed by culturepositive tissue for live spirochetes. Variations in the design included immediate application of the antibiotic at the site of the tick bite, delayed application at the site of tick bite, and application at a site distal to the tick bite. In the primary group (immediate application at the site of the tick bite), 0 of 12 mice in the azithromycin group were culture positive compared with 11 of 12 in the doxycycline group and 11 of 11 in the control group, resulting in $100 \%, 8 \%$, and $0 \%$ protection, respectively $(P<.001)$. Additionally, the $4 \%$ azithromycin cream provided $100 \%$ protection ( 0 of 10 culture positive) when applied at a site distal to the tick bite, $100 \%$ protection ( 0 of 12 culture positive) when applied at the tick bite site up to 3 days after tick removal, and $74 \%$ protection (3 of 12 culture positive) when applied at the tick bite site 14 days after tick removal.

This study suggests that $4 \%$ topical azithromycin cream offers $100 \%$ protection both directly and systemically for Lyme disease prophylaxis in a murine model within 3 days of exposure. The authors are hopeful this represents a future treatment option for Lyme disease prevention and that their research will prompt further studies with more test subjects, larger animals, and eventually human trials. Limitations of the study include only using a murine model, the pharmacological differences between azithromycin and doxycycline, and the small sample size.

(Antimicrob Agents Chemother. 2014;58:348-351) J Piesman, A Hojgaard, AJ Ullmann, MC Dolan.
Prepared by Cody Hood, MD, University of Utah Emergency Medicine Resident, Salt Lake City, UT, USA.

\section{BMJ OPEN}

Physiological Variables Associated with the Development of Acute Mountain Sickness at the South Pole

Exposure to altitudes greater than $2500 \mathrm{~m}$ (8202 feet) without acclimatization can result in acute mountain sickness (AMS) and is characterized by headache, malaise, fatigue, sleep disturbance, and gastrointestinal complaints. This prospective observational study included 98 participants from the US Antarctic Program who did not use prophylactic acetazolamide before rapidly ascending to the Amundsen-Scott South Pole Station ( 3200 $\mathrm{m})$ and was collected over 2 austral summer seasons in 20052006 and 2006-2007. Baseline questionnaires collected at McMurdo station (sea level) included the Lake Louise Symptom score and additional questions regarding physical symptoms related to AMS, medical history, current medications, lifestyle, exercise habits, and previous experience at high altitude. Age, sex, body mass index, blood pressure, heart rate, and oxygen saturation were also recorded. Blood draws were obtained at sea level 1 to 2 days before departure for the South Pole station. AMS symptom questionnaires were repeated by participants on the plane to Amundsen-Scott and for the first 7 days at $3200 \mathrm{~m}$. Blood draws were repeated on day 3 of arrival to altitude. Of the 98 participants, 30 experienced AMS, defined as a Lake Louise Symptom score of greater than or equal to 3 along with a concurrent headache. Between the groups of those who experienced AMS and those who did not, statistically significant differences were present in several parameters including heart rate, blood pressure, sodium concentration, low-density lipoproteins, eosinophils, tumor-necrosis factor alpha, vascular endothelial growth factor, and dopamine. The authors found that total body water regulation and inflammation are key factors in AMS development. Although further research is necessary, the authors suggest that maintaining intravascular volume to minimize edema combined with the use of anti-inflammatory medications could help prevent development of AMS.

(BMJ Open. 2013;3:e64) MF Harrison, P Anderson, A Miller, K O'Malley, M Richert, J Johnson, BD Johnson.

Prepared by Heather Beasley, University of Utah School of Medicine Medical Student, Salt Lake City, UT, USA.

\section{SCANDINAVIAN JOURNAL OF MEDI- CINE AND SCIENCE IN SPORTS}

Adherence of Backcountry Winter Recreationists to Avalanche Prevention and Safety Practices in Northern Italy

Backcountry recreationists account for approximately $47 \%$ of all annual avalanche fatalities in Europe. Although there are an 
increasing number of backcountry users, the annual mortality secondary to avalanches remains approximately the same. Despite efforts to increase avalanche education and awareness, accidents and fatalities continue to occur. The investigators conducted a comprehensive survey of skiers and snowshoers in the backcountry to assess avalanche prevention and safety practices.

A verbal questionnaire was administered at 22 backcountry access points during a 1-week period in the European Alps near South Tyrol, Italy. Data were collected from 1927 groups, and of the 5576 individuals who participated in the survey, $77.7 \%$ were skiers and $22.3 \%$ were snowshoers. Overall, a greater proportion of the skier group had read the avalanche bulletins compared with the snowshoer group (78.4\% vs $47 \%$ ), and the skier group better identified the correct avalanche danger for the day (52.5\% vs $28 \%)$. Additionally, skiers were much more likely to carry standard rescue equipment including transceiver, probe, and shovel when compared with snowshoers $(80.6 \%$ vs $13.7 \%$ ). Other factors associated with greater adherence to minimal advisable avalanche safety practices included younger age, more backcountry tours per season, traveling in a larger group, and starting earlier in the day. Individuals traveling alone were the least informed and prepared for backcountry travel.

The investigators defined "complete adherence" as having read the avalanche bulletin, being able to recall the danger rating, and carrying standard safety equipment. For the skier and snowshoer groups combined, there was only a $41.5 \%$ rate of complete adherence, whereas $36.5 \%$ had partial adherence and $21.9 \%$ had no adherence to standard safety recommendations. The authors identified an obvious need for further education of safe backcountry travel and avalanche awareness practices. Future studies should include transnational surveys to detect regional differences and correlating backcountry travel practices with morbidity and mortality.

(Scand J Med Sci Sports 2013 July [Epub ahead of print]) E Procter, G Strapazzon, T Dal Cappello, L Castlunger, HP Staffler, H Brugger.

Prepared by Wendy Hanna, MD, University of Utah Emergency Medicine Resident, Salt Lake City, UT, USA. 